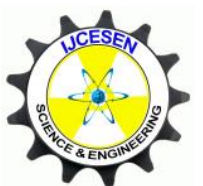

Copyright C IJCESEN
International Journal of Computational and

Experimental $\boldsymbol{S}$ cience and Engineering

(IJCESEN)

Vol. 5-No.2 (2019) pp. 56-60

http://dergipark.gov.tr/ijcesen

Research Article

\title{
Viscosity Change and its Effect on Pressure Distribution in Hydrodynamically Lubricated Journal Bearing
}

\author{
Iredia ERHUNMWUN ${ }^{1}$, John AKPOBI ${ }^{2}$ \\ ${ }^{1}$ University of Benin, Faculty of Engineering, Department of Production Engineering, Benin City, Nigeria \\ ${ }^{2}$ University of Benin, Faculty of Engineering, Department of Production Engineering, Benin City, Nigeria
}

* Iredia ERHUNMWUN : iredia.erhunmwun@ uniben.edu

ORCID: 0000-0002-0497-8220

\section{$\underline{\text { Article Info: }}$}

DOI: $10.22399 /$ ijcesen.530596

Received : 22 February 2019

Accepted : 27 March 2019

\section{$\underline{\text { Keywords }}$}

Reynolds Equation

Journal bearing

Weak formulation

Viscosity

Finite Element Method

\begin{abstract}
$\underline{\text { Abstract: }}$
In this work, a parametric study of the change in lubricant viscosity with pressure variation of fluid (lubricant) in a hydrodynamic journal bearing was carried out. This study was carried out using the Finite Element Method (FEM) and this was used to analyse the pressure behaviour of the bearing using the classical Reynolds Equation. From the result, it was observed that at the beginning of the bearing where the pressure was equal to the ambient pressure, the pressure increases thereafter till about $0.01875 \mathrm{~m}$ where the pressure was at maximum. From this point to the other trailing end of the bearing, the pressure decreases to the ambient pressure at $L=0.025 \mathrm{~m}$. At the early stage in the bearing, the pressure increase was seen not to be that significant. This was usually between the ranges of $0 \leq L \leq 0.00625 \mathrm{~m}$. From this point onward, the pressure begins to increase significantly. The reverse was the case when the bearing's angular displacement was in the range of $180^{\circ} \leq L \leq 360^{\circ}$. The result also shows a linear relationship between viscosity and pressure. The result obtained when compared with relevant literature shows a strong positive agreement.
\end{abstract}

\section{Introduction}

Journal bearings have been widely used for a long time but their lubrication principle was unknown until Reynolds derived his well-known Reynolds Equation [1]. The Reynolds Equation gave a basic relationship of the pressure of a convergent oil film in an eccentric bearing with the bearing parameters, such as the velocity, geometry and lubricant viscosity under a number of assumptions. Since then, extensive investigations have been carried out in this field by many researchers trying to obtain the pressure from the Reynolds Equation.

Sep in 2005 investigated the distributions of pressure, temperature and oil flow velocity of a journal bearing with a two-component surface layer [2]. Also, Mane and Soni in 2013 presented a paper on the 3D model of hydrodynamic plain journal bearing using COMSOL Multiphysics 4.3a software [3].
Again, Tom et al. in 2015, derive a novel and rigorous correction to the classical Reynolds lubrication approximation for fluids with viscosity depending upon the pressure. Their analysis shows that the pressure dependence of viscosity leads to additional nonlinear terms related to the shear-rate and arising from a non-negligible cross-film pressure [4].

Furthermore, Verma and Samant in 2016 carried out an unsteady transient analysis for hydrodynamically lubricated journal bearing with infinitely long approximation. Performance characteristics were investigated by oscillating the journal velocity as a 'sine' function of the angle for which the journal rotates with an angular speed. Results comprising of Minimum lubricant film thickness, dynamic pressure and load, wall shear stress, eccentricity, temperature distribution and heat flux with respect to time were presented in the 
analysis graphically with the aid of ORIGION PRO 8 [5].

Added to above, Hamdavi et al. in 2016 carried out theoretical studies and approach for finite fluid film journal bearing. In the research, the oil film pressure, load carrying capacity and attitude angle for plain and grooved short bearing were calculated and compared. Their result shows that applying one groove at inlet bearing surface, there was a decline in the performance of finite fluid film journal bearings [6].

Lastly, Shinde and Nagare in 2016 carried out an experimental evaluation of performance parameters (such as temperature, frictional torque and coefficient of friction) of journal bearing operating in boundary/ mixed lubrication regimes [7] and Manojkumar et al. analysed Elasto-hydrodynamic journal bearing using CFD and fluid structural interactions (FSI) approach [8].

In all the literatures reviewed so far, there was no general analytic solution to the classical Reynolds Equation that models the effect of side or end leakage [9]; however, approximate solutions have been obtained by using electrical analysis, mathematical summations, relaxation methods and numerical and graphical methods. This study is presented to investigate the effect of change in viscosity with the pressure distribution in a journal bearing under hydrodynamic lubrication assuming that the bearing in infinitely short.

\section{Governing Equation and boundary conditions}

The classical Reynolds equation is used to analyse the pressure distribution in an infinitely short hydrodynamic journal bearing is shown thus:

$$
\frac{\partial}{\partial z}\left(\frac{h^{3}}{\mu} \frac{\partial P}{\partial z}\right)=6 U \frac{\partial h}{\partial x}
$$

where $\mathrm{P}=$ pressure, $\mathrm{z}=$ Axial direction, $\mathrm{h}=$ Film Thickness, $\mu=$ Lubricant viscosity, $\mathrm{x}=$ Circumferential direction, $\mathrm{U}=$ Linear velocity

This Equation is subject to the following set of initial and boundary conditions.

$$
P(\theta, 0)=P(\theta, L)=0
$$

where $\theta=$ Angular displacement, $\mathrm{L}=$ Length of the bearing

For symmetry reasons,

$$
\left.\frac{\partial P}{\partial z}\right|_{z=0}=0
$$

The domain of the bearing consists of all points between $z=0$ and $z=L$ i.e. $\Omega=(0, L)$. The domain was divided into a set of line elements, a typical element being of length $h_{e}$ and located between two arbitrary points $\mathrm{A}$ and $\mathrm{B}$. The collection of such elements is called the finite element mesh of the domain. The reason for dividing the domain into finite elements was to represent the geometry of the domain and to approximate the solution over the entire domain.

\section{Mathematical analysis}

In the development of the weak form, we assumed a linear mesh and placed it over the domain. This was done by multiplying Eq. 1 by the weighted function (w) and integrating the final Equation over the domain. This results in the mathematical expression in Eq. 4.

$$
\int_{z_{A}}^{z_{B}} \frac{h^{3}}{\mu} \frac{\partial w}{\partial z} \frac{\partial P}{\partial z} d z+\int_{z_{A}}^{z_{R}} 6 w U \frac{\partial h}{\partial x} d z-w Q_{A}-w Q_{B}=0
$$

Eq. 4 is known as the weak form of the governing equation for hydrodynamic lubrication in journal bearing.

The weak form requires that the approximation chosen for $P$ should be at least linear in $z$ so that there are no terms in eq. 4 that are identically zero. Since the primary variable is simply the function itself, the Lagrange family of interpolation functions is admissible. We proposed that $P$ is the approximation over a typical finite element domain by the expression:

$$
P^{e}=\sum_{j=1}^{n} P_{j}^{e} \psi_{j}^{e}(z) \text { and } w=\psi_{i}^{e}(z) \quad i, j=1,2,3
$$

where $w=\psi_{i}^{e}(z)$ is the trial function

In Galerkin's weighted residual method, the weighting functions are chosen to be identical to the trial functions [10].

Substitute eq. 5 into eq. 4 , we have:

$$
\begin{aligned}
& \frac{h^{3}}{\mu}\left[K_{i j}^{e}\right]\left\{P_{j}^{e}\right\}=-6 U \frac{\partial h}{\partial x}\left\{F_{i}^{e}\right\}+\left\{Q_{i}^{e}\right\} \\
& \text { where } K_{i j}^{e}=\int_{z_{A}}^{z_{B}} \frac{\partial \psi_{i}^{e}(z)}{\partial z} \frac{\partial \psi_{j}^{e}(z)}{\partial z} d z
\end{aligned}
$$




$$
\begin{aligned}
& \text { assenfiphlimg, } \int_{z_{A}}^{z_{B}} w e^{e}(\bar{a}) d z \\
& Q_{i}^{e}=\psi_{i}^{e}(z) Q_{A}+\psi_{i}^{e}(z) Q_{B}
\end{aligned}
$$

Eq. 6 is referred to as the finite element based model, eq. 7 is known as the bearing matrix and eq. 8 is referred to as the wedge matrix.

Hence, the one-dimensional Lagrange quadratic interpolation function for Equation becomes

$$
\begin{gathered}
\psi_{1}=\left(1-\frac{z}{h_{e}}\right)\left(1-\frac{2 z}{h_{e}}\right), \\
\psi_{2}=\frac{4 z}{h_{e}}\left(1-\frac{z}{h_{e}}\right), \\
\psi_{3}=-\frac{z}{h_{e}}\left(1-\frac{2 z}{h_{e}}\right)
\end{gathered}
$$

where $h_{e}=$ Elemental length of the bearing

3.1. Evaluating the bearing matrix $\left\lfloor K_{i j}\right\rfloor$ and wedge matrix $\left\{F^{e}\right\}$ for journal bearing

To evaluate the $K_{i j}$ matrix, we substitute eq. 10 accordingly into eqs. 7 and 8 respectively, we have;

$$
\begin{aligned}
& K^{e}=\frac{1}{3 h_{e}^{3}}\left[\begin{array}{ccc}
7 h_{e}^{2}-24 h z_{e} z_{A}+48 z_{e}^{2} & -8\left(h_{e}^{2}-3 h_{e} z_{A}+12 z_{A}^{2}\right) & h_{e}^{2}+48 z_{A}^{2} \\
\left.h_{e}^{2}+48 z_{A}^{2} z_{A}^{2}+12 z^{2}\right) & 16\left(h_{e}^{2}+12 z_{A}^{2}\right) & -8\left(h_{e}^{2}+3 h_{e} z_{A}+12 z_{A}^{2}\right) \\
-8\left(h_{e}^{2}+3 h_{e} z_{A}+12 z_{A}^{2}\right) & 7 h_{e}^{2}+24 h_{e} z_{A}+48 z_{e}^{2}
\end{array}\right] \\
& F=\left\{\begin{array}{l}
\frac{h_{e}}{6}-z_{A}+\frac{2 z_{A}^{2}}{h_{e}} \\
\frac{2\left(h_{e}^{2}-6 z_{A}^{2}\right)}{3 h_{e}} \\
\frac{h_{e}}{6}+z_{A}+\frac{2 z_{A}^{2}}{h_{e}}
\end{array}\right\}
\end{aligned}
$$

Eq. 11 represents the generalized form of the bearing matrix for the entire domain of the bearing and eq. 12 it represents the generalized form of the wedge matrix for the entire domain of the bearing..

In our analysis, the bearing domain was divided into four quadratic elements as shown in Figure 1.

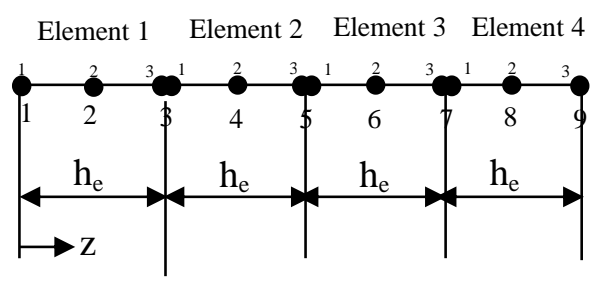

Figure 1: A four quadratic element
Analysing the elements part by part and finally

$\left[K^{e}\right]=\frac{1}{3 h_{e}}\left[\begin{array}{ccccccccc}7 & -8 & 1 & 0 & 0 & 0 & 0 & 0 & 0 \\ -8 & 16 & -8 & 0 & 0 & 0 & 0 & 0 & 0 \\ 1 & -8 & 38 & -80 & 49 & 0 & 0 & 0 & 0 \\ 0 & 0 & -80 & 208 & -128 & 0 & 0 & 0 & 0 \\ 0 & 0 & 49 & -128 & 230 & -344 & 193 & 0 & 0 \\ 0 & 0 & 0 & 0 & -344 & 784 & -440 & 0 & 0 \\ 0 & 0 & 0 & 0 & 193 & -440 & 614 & -800 & 433 \\ 0 & 0 & 0 & 0 & 0 & 0 & -800 & 1744 & -944 \\ 0 & 0 & 0 & 0 & 0 & 0 & 433 & -944 & 511\end{array}\right]$

$$
\left\{F^{e}\right\}=\frac{h_{e}}{6}\left\{\begin{array}{c}
1 \\
4 \\
8 \\
-20 \\
56 \\
-92 \\
152 \\
-212 \\
127
\end{array}\right\}
$$

Substitute in eq. 6, eqs. 13 and 14 and finally, substituting the boundary conditions in eqs. 2 and 3 and also these parameters $h_{e}=L / 4, U=\omega r$, $x=r \theta$ and $h=c(1+\varepsilon \cos \theta)$, we have:

$$
\left[\begin{array}{ccccccc}
16 & -8 & 0 & 0 & 0 & 0 & 0 \\
-8 & 38 & -80 & 49 & 0 & 0 & 0 \\
1 & -80 & 208 & -128 & 0 & 0 & 0 \\
0 & 49 & -128 & 230 & -344 & 193 & 0 \\
0 & 0 & 0 & -344 & 784 & -440 & 0 \\
0 & 0 & 0 & 193 & -440 & 614 & -800 \\
0 & 0 & 0 & 0 & 0 & -800 & 1744
\end{array}\right]\left\{\begin{array}{l}
P_{2} \\
P_{3} \\
P_{4} \\
P_{5} \\
P_{6} \\
P_{7} \\
P_{8}
\end{array}\right\}=\frac{3 \omega L^{2} \mu \varepsilon \sin \theta}{16 c^{2}(1+\varepsilon \cos \theta)^{3}}\left\{\begin{array}{c}
4 \\
8 \\
-20 \\
56 \\
-92 \\
152 \\
-212
\end{array}\right\}
$$

where $\mathrm{L}=$ Length of Bearing, $\mathrm{U}=$ Linear velocity, $\omega=$ Angular velocity, $\mathrm{r}=$ radius of bearing, $\mathrm{c}=$ radial clearance, $\varepsilon=$ eccentricity ratio

\section{Results and Discussion}

The parameters used in this analysis is as shown in Table 1.

Table 1: Parameters for calculation for short journal bearing [3]

\begin{tabular}{|c|c|}
\hline Parameters & Short bearing \\
\hline Lengths of bearing & $2.50 \times 10^{-2}(\mathrm{~m})$ \\
\hline Diameter of journal & $0.05(\mathrm{~m})$ \\
\hline Radial clearance & $2.50 \times 10^{-5}(\mathrm{~m})$ \\
\hline Eccentricity & $1.25 \times 10^{-5}(\mathrm{~m})$ \\
\hline Eccentricity ratio & 0.50 \\
\hline Speed of journal & $1000(\mathrm{rpm})$ \\
\hline Dynamic viscosity of oil & $0.19(\mathrm{~Pa} . \mathrm{S})$ \\
\hline Inlet temperature & $315(\mathrm{~K})$ \\
\hline
\end{tabular}

4.1. Variation of pressure with axial displacements for journal bearing 
It was observed that at the beginning of the bearing where the pressure was equal to the ambient pressure, the pressure increases thereafter till about $0.01875 \mathrm{~m}$ where the pressure was at maximum. From this point to the other trailing end of the bearing, the pressure decreases to the ambient pressure at $\mathrm{L}=0.025 \mathrm{~m}$. This was shown in Figure 2. At the early stage in the bearing, the pressure increase was seen not to be that significant. This was usually between the ranges of $0 \leq L \leq 0.00625 m$. From this point onward, the pressure begins to increase significantly. The reverse was the case when the bearing's angular displacement was in the range of $180^{\circ} \leq \theta \leq 360^{\circ}$

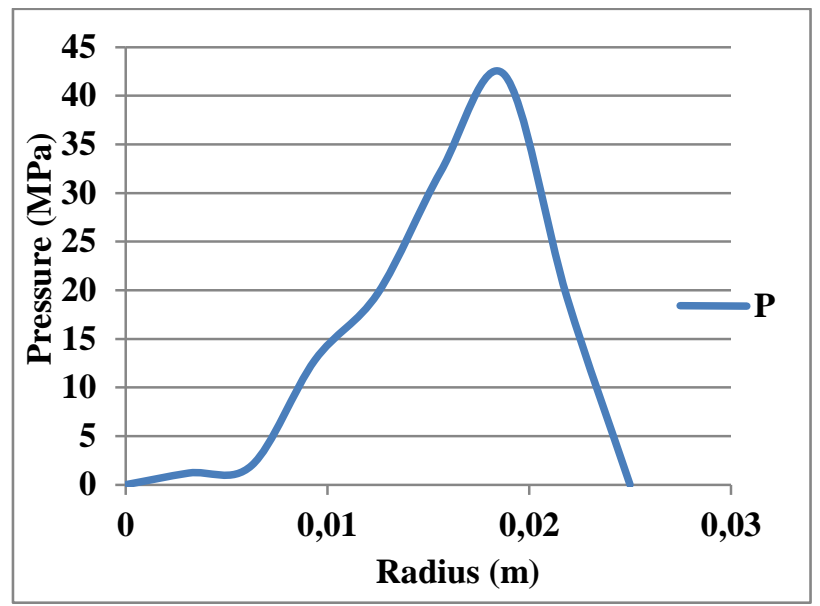

Figure 2. Variation of pressure against axial displacement

The result from FEM was validated by comparing it with the result from the analytical solution and the result published by Mane and Soni in 2013 [3].

Table 2: Comparison between FEM, COSMOL and Analytical solution

\begin{tabular}{|c|c|c|c|}
\hline $\mathrm{Q}\left(^{0}\right)$ & ANALYTICAL & COSMOL & FEM \\
\hline 0 & 0 & 0 & 0 \\
\hline 30 & 122565 & 120231 & 122000 \\
\hline 60 & 317565 & 315456 & 318375 \\
\hline 90 & 718245 & 717546 & 718023 \\
\hline 120 & 1491345 & 1415657 & 1473959 \\
\hline 150 & 2003459 & 1954563 & 1969648 \\
\hline 180 & 0 & 0 & 0 \\
\hline 210 & -2003459 & -1954563 & -1969648 \\
\hline 240 & -1491345 & -1415657 & -1473959 \\
\hline 270 & -718245 & -717546 & -718023 \\
\hline 300 & -317565 & -315456 & -318375 \\
\hline 330 & -121565 & -120231 & -122000 \\
\hline 360 & 0 & 0 & 0 \\
\hline
\end{tabular}

The comparison shows a strong positive correlation. The FEM approximates the solution to the analytical solution better than the result by [3]. This is shown in Table 2.

\subsection{Pressure variation with change in lubricant viscosity}

When a bearing begins to rotate, the temperature of the fluid between the journal and the bearing begins to increase. This increase in temperature affects the viscosity of the fluid (lubricant). Therefore, it is very important to know how the change in the viscosity of the lubricant affects the pressure distribution in the bearing. From this analysis, it was observed that as the viscosity of the fluid in the hydrodynamic journal bearing increases, the pressure of the fluid in the bearing also increases. The result obtained show that the change in the lubricant viscosity varies linearly with the change in pressure.

Therefore, in the design of journal bearings, the lubricant used should have low viscosity so as to reduce the pressure in the bearing. The graph showing the variation of pressure with viscosity is shown in Figure 3.

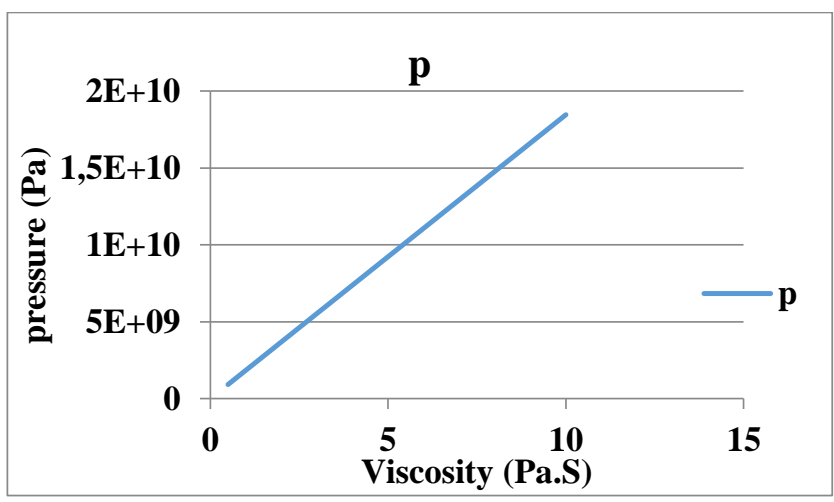

Figure 3. Variation of pressure with lubricant viscosity

\section{Conclusion}

The pressure distribution of the hydrodynamic journal bearing lubricated with oil under steady state and constant temperature consideration has been analysed. Based on the results and discussion presented in the preceding section, the following conclusions can be reached.

The Finite Element Method (FEM) form of the general governing Reynolds Equation was derived and implemented for hydrodynamic journal bearing. Using Reynolds Equation, a numerical solution has been developed for short journal bearing under steady state. 
The change of pressure with lubricant viscosity was also investigated and it was shown that there was a linear relationship between the lubricant viscosity and the pressure in the bearing.

\section{References}

[1] O. Reynolds. On the theory of lubrication and its application to Mr. Beauchamps Tower's Experiments, including an experimental determination of the viscosity of olive oil. Philosophical Transactions of the Royal Society London, 177, pp. 157-23, 1886.

[2] J. Sep. Three dimensional hydrodynamic analysis of a journal bearing with a two component surface layer. Tribology International, 38, pp. 97-104, 2005.

[3] R.M. Mane and S. Soni. Analysis of Hydrodynamic Plain Journal Bearing. Expert from the proceedings of the 2013 COSMOL conference in Bangalore.

[4] G. Tom, K.R. Rajagopal, S. Rolf, and V. Juha. Nonlinear Reynolds equation for hydro dynamic lubrication. 39, pp. 5299-5309, 2015.

[5] A. Verma, and S.S. Samant. Inspection of Hydrodynamic Lubrication in Infinitely Long Journal Bearing with Oscillating Journal Velocity. Journal of Applied Mechanical Engineering, 5, pp. 1-7, 2016.

[6] S. Hamdavi, H.H. Ya, T.V.V.L.N. Rao, and K.M. Faez. An analytical approach to investigate the effect of grooved surface on short journal bearing's performance. ARPN Journal of Engineering and Applied Science, 11(20), pp. 12045-12049, 2016.

[7] P.D. Shinde and P.N. Nagare. Experimental Evaluation of Performance Parameters of Journal Bearing Operating in Boundary/ Mixed Lubrication Regimes. International Advanced Research Journal in Science, Engineering and Technology, 2nd International Conference in Advance Mechanical Engineering (ICAME-2016), 3(1), pp. 116-120, 2016.

[8] Manojkumar, Shamburaje and Rameshwar. CFD analysis of elasto hydro-dynamic lubrication journal bearing using castor oil and bronze material. International Journal of Advance Research and Innovative Ideas in Engineering, 2(2), pp. 56-67, 2016.

[9] Budynas-Nisbett, Shigley’s Mechanical Engineering Design, Eighth Edition, McGraw-Hill, 2008.

[10] J.N. Reddy, Introduction to the Finite Element Method, Second edition, McGraw-Hill series in Mechanical Engineering. 1993. 